\title{
A!
}

This is an electronic reprint of the original article.

This reprint may differ from the original in pagination and typographic detail.

Moilanen, Antti J.; Daskalakis, Konstantinos S.; Taskinen, Jani M.; Törmä, Päivi

\section{Spatial and Temporal Coherence in Strongly Coupled Plasmonic Bose-Einstein Condensates}

Published in:

Physical Review Letters

DOI:

10.1103/PhysRevLett.127.255301

Published: 15/12/2021

Document Version

Publisher's PDF, also known as Version of record

Please cite the original version:

Moilanen, A. J., Daskalakis, K. S., Taskinen, J. M., \& Törmä, P. (2021). Spatial and Temporal Coherence in Strongly Coupled Plasmonic Bose-Einstein Condensates. Physical Review Letters, 127(25), 1-7. [255301]. https://doi.org/10.1103/PhysRevLett.127.255301

This material is protected by copyright and other intellectual property rights, and duplication or sale of all or part of any of the repository collections is not permitted, except that material may be duplicated by you for your research use or educational purposes in electronic or print form. You must obtain permission for any other use. Electronic or print copies may not be offered, whether for sale or otherwise to anyone who is not an authorised user. 


\title{
Spatial and Temporal Coherence in Strongly Coupled Plasmonic Bose-Einstein Condensates
}

\author{
Antti J. Moilanen $\odot,{ }^{1}$ Konstantinos S. Daskalakis $\odot,{ }^{1,2}$ Jani M. Taskinen, ${ }^{1}$ and Päivi Törmä ${ }^{1, *}$ \\ ${ }^{1}$ Department of Applied Physics, Aalto University School of Science, P.O. Box 15100, Aalto FI-00076, Finland \\ ${ }^{2}$ Department of Mechanical and Materials Engineering, Turku University Faculty of Technology, Turku FI-20014, Finland
}

(Received 19 March 2021; accepted 22 November 2021; published 15 December 2021)

\begin{abstract}
We report first-order spatial and temporal correlations in strongly coupled plasmonic Bose-Einstein condensates. The condensate is large, more than 20 times the spatial coherence length of the polaritons in the uncondensed system and 100 times the healing length, making plasmonic lattices an attractive platform for studying long-range spatial correlations in two dimensions. We find that both spatial and temporal coherence display nonexponential decay; the results suggest power-law or stretched exponential behavior with different exponents for spatial and temporal correlation decays.
\end{abstract}

DOI: 10.1103/PhysRevLett.127.255301

Three-dimensional Bose-Einstein condensates (BECs) in thermal equilibrium exhibit long-range order of spatial correlations that, in principle, extend to infinity. In 2D, true long-range order is prohibited by thermal fluctuations [1-3]. Nevertheless, it has been shown that quasilong-range order may persist in equilibrium systems through the Berezinskii-Kosterlitz-Thouless (BKT) transition $[4,5]$ and in nonequilibrium via the dynamical phase ordering of Kardar-Parisi-Zhang (KPZ) [6]. Exciton polaritons (photon-exciton quasiparticles) offer a platform for studying correlations of driven-dissipative condensates in $2 \mathrm{D}$, yet the occurrence of long-range order in these systems has remained elusive. Typically the systems have been too small to give definitive answers about decay at long distances. We introduce strongly coupled plasmonic BECs as a system for studying correlations: we demonstrate a condensate of size more than 20 times the spatial coherence length of the polaritons and 2 orders of magnitude larger than the healing length. In previous reports of exciton-polariton condensates and photon BECs, the ratio of condensate size to length scales such as de Broglie wavelength or healing length has been approximately a factor of 10 at best [7-10]. We find decay of spatial and temporal coherence that is clearly nonexponential; the results are best described by power-law or stretched exponential decay with different exponents for spatial and temporal correlations.

Various scenarios of long-range correlation decay have been predicted for 2D systems. In equilibrium BECs, thermal fluctuations give rise to vortices and antivortices that disrupt the long-range order. In the BKT transition, below a critical temperature, vortices and antivortices are paired such that their phases cancel out allowing algebraic decay of correlations, $g^{(1)}(x) \propto x^{-b}$. The BKT transition entails power-law decays of both spatial and temporal correlations with equal exponents $b_{s}=b_{t} \leq 0.25$ [11], although in the presence of drive and dissipation the exponents may differ as $b_{s}=2 b_{t}$ $[12,13]$. In nonequilibrium condensates, the occurrence of the BKT transition has been theoretically both supported $[12,14]$ and refuted [15]. Long-range phase ordering could be restored by the KPZ mechanism with correlations decaying as a stretched exponential [15-17]. A crossover between the KPZ dynamics and equilibriumlike BKT has been proposed, determined by the degree of anisotropy in the system $[15,18]$. Contrary to BEC, for a usual laser, an exponential decay of temporal correlations is expected [19].

Semiconductor polariton condensates [20-22] are typically far from equilibrium conditions due to the short lifetime of polaritons with respect to their thermalization time, however, a quasiequilibrium state can be achieved by a dynamical balance of pump and dissipation [23,24]; nearequilibrium conditions are also possible [7,25]. Polaritons decay via emission of photons, providing optical access to the properties of the condensate. This makes polariton condensates an attractive platform for studying spatial and temporal correlations. Early reports on spatial correlations in microcavity polariton condensates indicated algebraic decay $[26,27]$, whereas temporal correlations have been shown to decay exponentially or as a Gaussian [8,28-32]. There have been few studies governing both spatial and temporal correlations, thus the question of whether true long-range order exists in nonequilibrium polariton condensates remains open. At equilibriumlike conditions, with polariton lifetime exceeding the other timescales of the system, a BKT transition has been suggested with both spatial and temporal correlations showing a power-law decay with $b<0.25$ [7]. In general, establishing power-law behavior quantitatively is challenging [33].

Spatial and temporal correlations have been studied also in other luminous condensates. In photon BECs $[9,10]$ and plasmonic polariton condensates (polariton lasers) [34], correlations have shown exponential and Gaussian decays; 
the absence of long-range order has been attributed to small condensate size and finite-size effects [9] and to the presence of drive and dissipation [34]. Indeed, one of the central factors hindering the studies of long-range order in polariton and photon condensates has been the small system size $[15,17]$.

Here, we introduce a plasmonic polariton BEC with long-range spatial correlations that extend to remarkably long distances [35]. We present the first measurement and in-depth analysis of both spatial and temporal coherence in plasmonic BECs. Our results clearly show that the correlations differ both from a nonordered phase and from a laser. The exponents extracted by power-law and stretched exponential fits hint to a scenario between quasiequilibrium and nonequilibrium.

System and experiment.-Plasmonic lattices, comprising nanoparticle arrays covered with fluorescent molecules, have been used to create BECs in the weak [42] and strong [41] coupling regimes. Nanoparticle arrays give rise to surface lattice resonances (SLRs) which are hybrid modes of localized surface plasmon resonances in the individual nanoparticles and light diffracted to the periodic array [43-45]. The $\Gamma$ point of the SLR dispersion provides a band edge for lasing $[46,47]$ and condensation [41,42]. To study spatial correlation decay at long distances, we use structures similar to those in our previous work [41], but extend the system in $x$ to $500 \mu \mathrm{m}$, while keeping the $y$ dimension at $100 \mu \mathrm{m}$. The arrays are covered with an $80 \mathrm{mM}$ solution of IR-792 dye leading to strong coupling between the molecules and the SLR modes, which persists also at high pump fluences [36]. The plasmonexciton polaritons, called polaritons hereafter, in the system are bosonic quasiparticles consisting of light diffracted to the array, electron plasma oscillation, and the dye excitons. The molecules are excited by a pulsed laser $(50 \mathrm{fs}, 1 \mathrm{kHz}$, $800 \mathrm{~nm} / 1.55 \mathrm{eV}, 0.04 \mathrm{eV}$ FWHM) with a spot larger than the arrays. See the Supplemental Material [36] for descriptions of the samples and the experimental setup.

As in our previous work [41], upon increasing pump fluence, the sample luminescence exhibits a double threshold, where the first threshold corresponds to polariton lasing and the second to polariton BEC [Fig. 2(a)]. Distinct from most polariton condensates, we observe a thermal distribution that extends over a range of $2 k_{B} T$ at room temperature, see Fig. 2(b). Since the polariton lifetime without gain $\sim 100 \mathrm{fs}$ is on the same order as the other timescales of the system, it is reasonable to assume the condensate to be in a quasi- or nonequilibrium regime [48]. We observe a prominent change in the coherence properties between the lasing and BEC regimes.

We measure spatial and temporal coherence by a Michelson interferometer in a mirror-retroreflector configuration; a schematic of the experiment is presented in Fig. 1. The interference fringe contrast is directly proportional to the first-order correlation function between two points separated by $\left|\mathbf{r}-\mathbf{r}^{\prime}\right|, g^{(1)}\left(\mathbf{r}, \mathbf{r}^{\prime} ; \tau\right)$, where $\tau$ is the

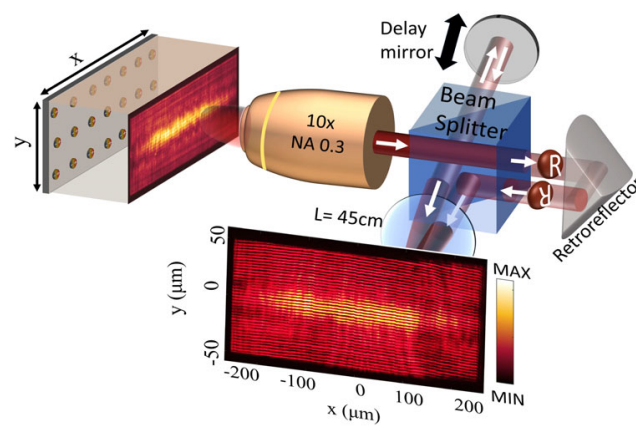

FIG. 1. Schematic of the experiment. The sample luminescence is imaged through a microscope to a Michelson interferometer, where the real-space image is inverted over both $x$ and $y$ and overlapped with the original image.

time delay between the interferometer arms. Thus, changing the longitudinal position of the mirror and recording interferograms over a range of delays allows for directly probing the temporal coherence. For spatial coherence, we measure a series of interferograms at fixed intervals around $\tau=0$ over three periods of light frequency oscillation. The amplitude and phase of the interference fringes are obtained by fitting a sinusoidal function through each pixel of the stack of normalized interferograms; see Supplemental Material [36] for details.

Spatial coherence.-Figure 2 shows interferograms, the corresponding $g^{(1)}(\mathbf{r},-\mathbf{r} ; \tau=0)$ maps, and average $g^{(1)}(x)$ along the long axis of the lattice for three excitation regimes: below, at, and above the BEC threshold. The phase maps of the fringes are presented in Fig. S4 of the Supplemental Material [36]. Below the threshold, spatial coherence emerges around the center of the array and decays toward the edges. At the threshold, coherence extends over a longer distance but gradual decay is visible. Remarkably, above the threshold, coherence is nearly constant throughout the array.

Let us have a closer look at the decay of correlations. Figures 3(a)-3(c) show $g^{(1)}$ as a function of radial separation of centrosymmetric points, $\Delta r=\left|\mathbf{r}-\mathbf{r}^{\prime}\right|$, for the pump fluences highlighted with filled symbols in Fig. 3(d). The measured $g^{(1)}(\Delta r)$ are fit to Gaussian, exponential, stretched exponential, and power-law functions: $g^{(1)}(\Delta r)=$ $a \exp \left[-(\Delta r / d)^{\beta}\right]$ and $g^{(1)}(\Delta r)=a(\Delta r)^{-b}$, where $\beta=2$ gives a Gaussian and $\beta=1$ an exponential function, and $0<a \leq 1$ is a scaling parameter. Power-law or stretched exponential behavior are expected to occur only above the $\mathrm{BEC}$ threshold. We exclude the short-range regime around the autocorrelation point $(\Delta r=0)$ from the fits, determined by the spatial coherence length of the polaritons in the uncondensed system (below the first threshold, $22 \mu \mathrm{m}$ [36]). All the fits are performed using the same fit range, and the best-fitting models are shown in Figs. 3(a)-3(c). At pump fluences below the BEC threshold, as shown in Figs. 3(a) and 3(b), spatial correlations decay as a Gaussian. Above the 

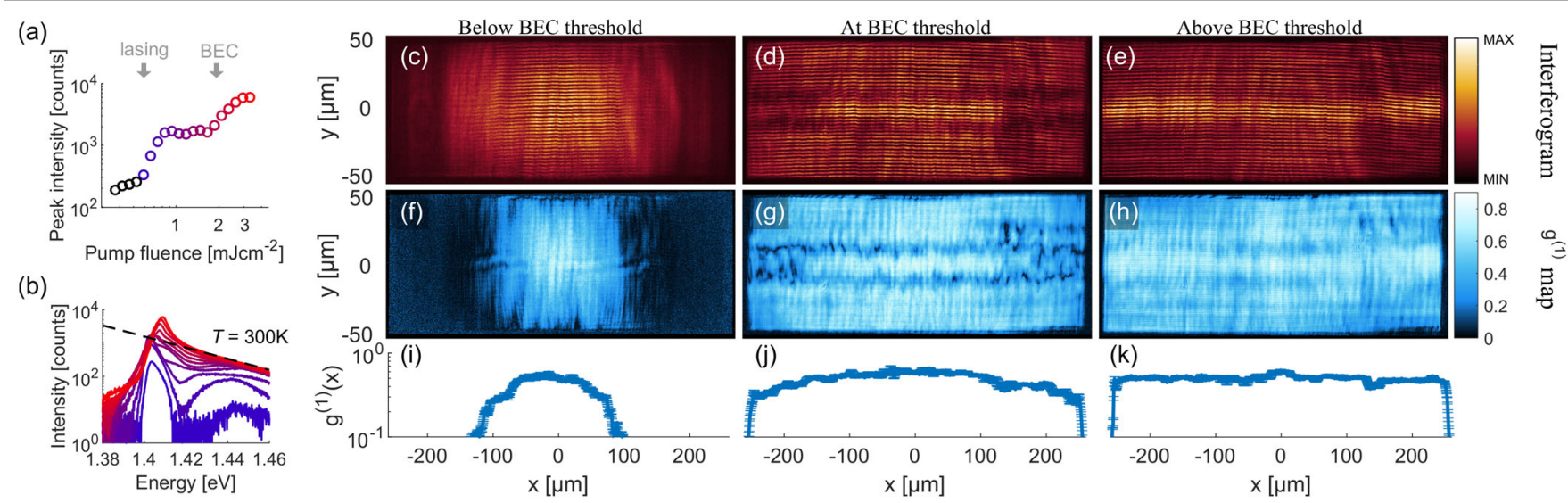

FIG. 2. Interferograms and spatial correlation maps. (a) Threshold curve and (b) photoluminescence spectra. The pump fluences are

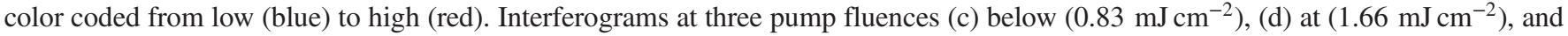
(e) above $\left(3.31 \mathrm{~mJ} \mathrm{~cm}^{-2}\right)$ the BEC threshold. (f)-(h) Maps of $g^{(1)}(\mathbf{r},-\mathbf{r})$ for the corresponding pump fluences. (i)-(k) Average $g^{(1)}(x)$ over the $y$ axis of the array. The error bars show the standard deviation of three measurements. A sample with random distribution of nanoparticles did not show coherence (Fig. S10 [36]).

threshold, Fig. 3(c), long-range spatial coherence covering the entire array emerges and the correlation function is nearly constant over a remarkably long distance: a fit to an

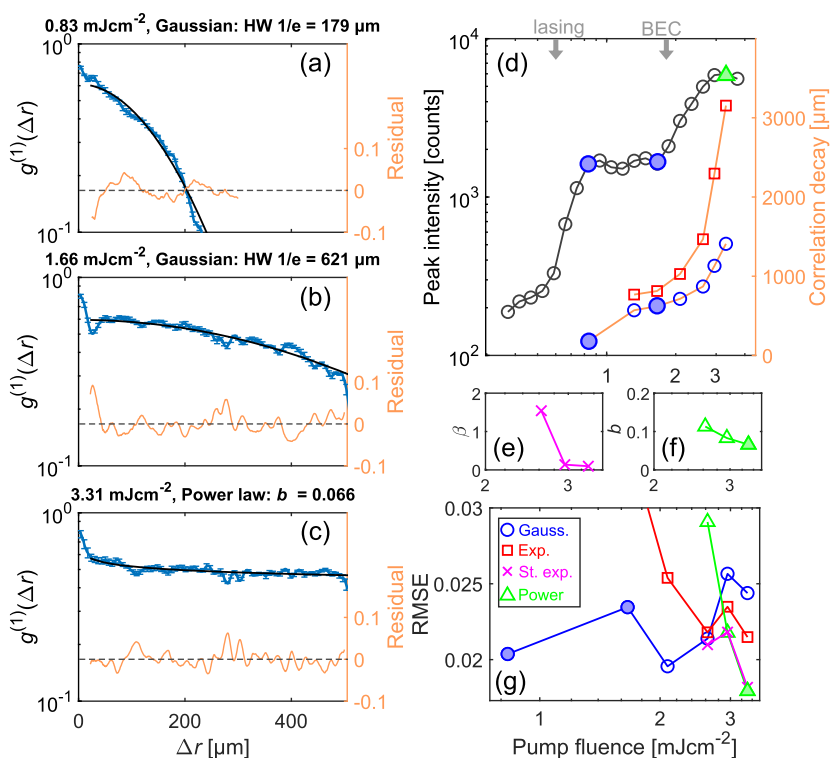

FIG. 3. Quantitative analysis of spatial correlation decay. Measured $g^{(1)}(\Delta r)$ at three pump fluences (a) below, (b) at, and (c) above the BEC threshold. The error bars represent the standard deviation of three measurements. The best-fitting functions are shown by black solid lines. (d) Threshold curve (black circles) and spatial correlation decay extracted from Gaussian (blue circles) and exponential fits (red squares). The fluences corresponding to (a)-(c) are highlighted with filled symbols. For the exponential function, the reported length is the decay constant and for the Gaussian the half-width (HW) at 1/e decay. Exponents extracted from (e) stretched exponential and (f) power-law fits. (g) RMSE of the fits. In (d)-(f) the horizontal axis values are in the same units as in $(\mathrm{g})$. exponential yields a decay length of more than $3000 \mu \mathrm{m}$, which is 6 times the long axis of the system and 2 orders of magnitude larger than the coherence length of the polaritons. The decay lengths given by Gaussian and exponential fits are presented in Fig. 3(d) alongside the threshold curve. However, the data are best fit to a power-law function with a small exponent of 0.07 and almost equally to a stretched exponential with an exponent of around 0.1. The fits to all four functions are presented in Fig. S8 of the Supplemental Material [36]. Figures 3(e) and 3(f) show the exponents obtained from the stretched exponential and power-law fits. The root-mean-square error (RMSE) of the fits are compared in Fig. 3(g).

The thermalization in the plasmonic polariton BEC involves stimulated processes [41]. Here, we separated the thermal tail (the part of the BEC spectrum that displays Maxwell-Boltzmann distribution) of the condensate emission and found that there seems to be some coherence present in the emission (Fig. S7 [36]), suggesting such experiments can be used for future studies of the thermalization process.

Temporal coherence.-Temporal coherence for increasing pump fluence is displayed in Fig. 4(d). The measured $g^{(1)}(|\tau|)$ are again fit to Gaussian, exponential, stretched exponential, and power-law functions, and the best-fitting models are shown in Figs. 4(a)-4(c). The short-range correlations, between $\tau=0$ and the temporal coherence of the polaritons in the uncondensed system (below the first threshold, $\tau=104 \mathrm{fs}$ ), are excluded from the fits [36]. Below the BEC threshold, as shown in Fig. 4(a), correlations decay exponentially with a decay constant of 366 fs. This already exceeds the temporal coherence of the polaritons due to the lasing triggered at the first threshold. Above the BEC threshold, Figs. 4(b) and 4(c), the data are best fit to a powerlaw function with an exponent of around 0.7-0.8; the stretched exponential fits almost equally with an exponent 


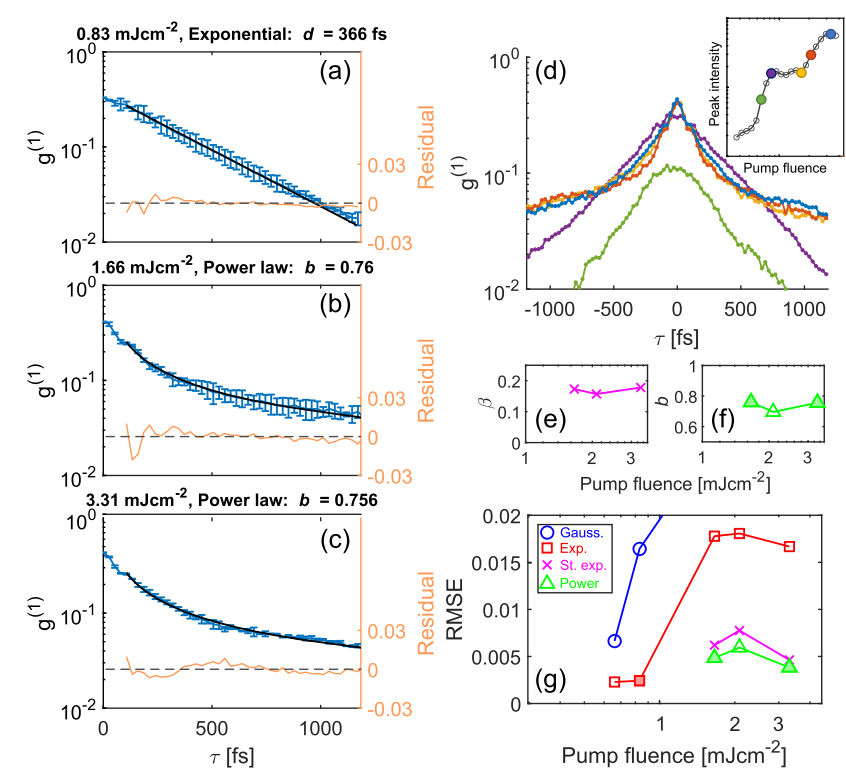

FIG. 4. Quantitative analysis of temporal correlation decay. (a)-(c) Measured $g^{(1)}(|\tau|)$ at three pump fluences (a) below, (b) at, and (c) above the BEC threshold. The error bars represent the standard deviation of the averaged $\tau<0$ and $\tau>0$ values from (d). Fits to the best-fitting function are shown with black solid lines. (d) Measured temporal coherence for different pump fluences, color coded according to the filled circles in the threshold curve (inset). Exponents extracted from (e) stretched exponential and (f) power-law fits. (g) RMSE of the fits.

of $\beta \sim 0.2$, given by Figs. 4(e) and 4(f). Figure 4(g) presents the RMSE of the fits. The decay of temporal coherence in the $\mathrm{BEC}$ regime is significantly slower than in the lasing regime (see also Fig. S11 of the Supplemental Material [36]).

The measured spatial coherence at $\Delta r$ is, in general, higher than the temporal one at $|\tau|=\Delta r / c$ ( $c$ is the speed of light), $g^{(1)}(\Delta r) \gg g^{(1)}(\Delta r / c)$, for instance, for $\Delta r=300 \mu \mathrm{m} 10$ times higher. This raises a concern about causality, needed for spontaneous formation of spatial coherence. However, this difference might be reduced by taking into account the different value of the two coherences, at zero distance and time, and the finite photoluminescence duration (Fig. S12 [36]). Based on Fig. S12, the duration of the output pulse is likely to be about 2 ps or longer.

Discussion.-At pump fluences far below the BEC threshold, but above the lasing threshold, the system behaves much like a polariton laser: spatial correlations decay as a Gaussian and temporal correlations as an exponential function. Nanoparticle array lasers, at weak coupling regime, have previously shown Gaussian decay of both spatial and temporal correlations [49], and at strong coupling, an exponential decay of spatial and a quasi-Gaussian decay of temporal correlations [34]. In polariton condensates, temporal correlation decay has been reported to be Gaussian $[8,28-30,50]$ or of Kubo form, i.e., Gaussian or exponential depending on whether the number fluctuations are slow or fast $[31,32]$.
Above the BEC threshold, both spatial and temporal correlations show decay that indicates power-law behavior. Spatial correlations decay with a small exponent of 0.07 , whereas temporal correlations decay with a large exponent of around 0.7-0.8. This clearly differs from equilibrium BKT transition, as could be expected due to the driven-dissipative nature of our system. It is worthwhile to mention that we have also looked at single-shot interferograms of the condensate and have not observed vortices. In driven-dissipative condensates, the power-law exponent may exceed the equilibrium BKT limit 0.25 , but our findings do not directly match with the reported experimental [26] or theoretical values [12,14].

A stretched exponential function, related to KPZ dynamics, fits approximately equally well as the power law, yielding small exponents $\beta$. The KPZ scaling of correlations has been mostly considered in the optical parametric oscillator regime of microcavity polariton condensates $[16,18]$. In the strongly anisotropic case, the KPZ equation leads to a power-law decay of spatial correlations with exponent $b_{s}=2 \chi$, where $\chi$ is the roughness exponent that in 2D takes the universal value of 0.39 [51-53]. Likewise, the temporal correlations decay as power law, but with an exponent that is $1 / 2$ of the spatial exponent, $b_{t}=\chi$. In the regime of weak anisotropy, the $2 \mathrm{D} \mathrm{KPZ}$ equation predicts a stretched exponential decay with $\beta_{s}=2 \chi=0.78$ for spatial and $\beta_{t}=2 \chi /(2-\chi)=0.48$ for temporal correlations [18]. Our results yield $\beta_{s} \approx 0.1$ and $\beta_{t} \approx 0.2$ above the BEC threshold.

Our system is not isotropic. The nanoparticles host dipolar and multipolar charge oscillation modes associated with directional radiation, which combined with the periodicity of the array in the $x$ and $y$ directions leads to SLR modes that are not isotropic in the plane. In an infinite or square array, there is still $x-y$ symmetry, but even this can be broken by the pump polarization (see the Supplemental Material [36] for further discussion). Evaluating the level of anisotropy in the sense of the theoretical predictions is difficult since our system is not analogous to those considered in Refs. [15,18], however, the KPZ scaling is expected to be rather universal.

Finally, it should be noted that the differences (e.g., RMSE) between fits to different models have typically been marginal, and therefore, establishing power-law behavior quantitatively is not straightforward [7,18,26,27]. Ideally the data should range over at least two decades to rigorously claim power-law behavior [33]. The results depend on the fit range, so it is of key importance to justify the range (short vs long range) deliberately. Another remark is that the regime of pump powers where the transition to a BKT phase or KPZ dynamics is expected could be very narrow $[12,14,17]$.

Summary and outlook.-We have characterized for the first time the spatial and temporal first-order correlations in a plasmonic BEC. We proposed plasmonic lattice 
condensates as a platform for studying long-range order in 2D condensates of light, with versatile system design and open-cavity character. The combination of large size and short cavity lifetime makes the system amenable for probing nonequilibrium BKT transition or KPZ dynamics $[15,17,18,54]$.

In this Letter, we have shown that the decay of correlations above the BEC threshold is nonexponential, in stark contrast to the nonordered and lasing phases. Our results indicate algebraic decay of both spatial and temporal correlations with different exponents; however, the stretched exponential fits approximately equally well. The exponents found do not quantitatively match with reported predictions for equilibrium or nonequilibrium systems. Although the scaling laws predicted by the equilibrium BKT and the nonequilibrium KPZ mechanisms might be universal, understanding the role of strongly coupled photonic, electronic, and vibrational states in the condensate formation is an important general question that requires future theory work and may bring in new physics. Moreover, the known plasmonic nanoparticle array mode structure $[43-45,55,56]$ should be incorporated in the theoretical analysis to quantify the degree of anisotropy.

Beyond fundamental studies of correlations, the plasmonic polariton BEC provides spatial coherence decay on the millimeter scale, which is 1-2 orders of magnitude larger than in other polariton or photon condensates before. Millimeter-scale spatial coherence has been previously reported in weakly coupled plasmonic lasers [49]. Strongly coupled plasmonic BECs provide also effective interactions [41], which may give rise to phenomena not accessible in weakly coupled and noninteracting systems, such as superfluidity [57]. The large extent of spatial coherence could be utilized in, for instance, on-chip applications for sensing, where lasing and condensation can make tiny effects observable.

We thank Jonathan Keeling and Kristin B. Arnardottir for their comments on the early version of the Letter. A. J. M. and P. T. acknowledge support by the Academy of Finland under Projects No. 303351, No. 327293, No. 318937 (PROFI), and No. 320167 [Flagship Programme Photonics Research and Innovation (PREIN)]. A. J. M. acknowledges financial support by the Jenny and Antti Wihuri Foundation. K. D. acknowledges financial support from the European Research Council (ERC) under the European Union's Horizon 2020 Research and Innovation Programme [Grant Agreement No. (948260)].

*paivi.torma@aalto.fi

[1] N. D. Mermin and H. Wagner, Absence of Ferromagnetism or Antiferromagnetism in One- or Two-Dimensional Isotropic Heisenberg Models, Phys. Rev. Lett. 17, 1133 (1966).

[2] P. C. Hohenberg, Existence of long-range order in one and two dimensions, Phys. Rev. 158, 383 (1967).
[3] I. Bloch, J. Dalibard, and W. Zwerger, Many-body physics with ultracold gases, Rev. Mod. Phys. 80, 885 (2008).

[4] J. M. Kosterlitz and D. J. Thouless, Ordering, metastability and phase transitions in two-dimensional systems, J. Phys. C 6, 1181 (1973).

[5] V. L. Berezinskii, Destruction of long-range order in one-dimensional and two-dimensional systems having a continuous symmetry group I. Classical systems, Sov. Phys. JETP 32, 493 (1971).

[6] M. Kardar, G. Parisi, and Y.-C. Zhang, Dynamic Scaling of Growing Interfaces, Phys. Rev. Lett. 56, 889 (1986).

[7] D. Caputo, D. Ballarini, G. Dagvadorj, C. Sánchez Muñoz, M. De Giorgi, L. Dominici, K. West, L. N. Pfeiffer, G. Gigli, F. P. Laussy, M. H. Szymanska, and D. Sanvitto, Topological order and thermal equilibrium in polariton condensates, Nat. Mater. 17, 145 (2018).

[8] K. S. Daskalakis, S. A. Maier, and S. Kena-Cohen, Spatial Coherence and Stability in a Disordered Organic Polariton Condensate, Phys. Rev. Lett. 115, 035301 (2015).

[9] T. Damm, D. Dung, F. Vewinger, M. Weitz, and J. Schmitt, First-order spatial coherence measurements in a thermalized two-dimensional photonic quantum gas, Nat. Commun. 8, 158 (2017).

[10] J. Marelic, L. F. Zajiczek, H. J. Hesten, K. H. Leung, E. Y. X. Ong, F. Mintert, and R. A. Nyman, Spatiotemporal coherence of non-equilibrium multimode photon condensates, New J. Phys. 18, 103012 (2016).

[11] D. R. Nelson and J. M. Kosterlitz, Universal Jump in the Superfluid Density of Two-Dimensional Superfluids, Phys. Rev. Lett. 39, 1201 (1977).

[12] P. Comaron, I. Carusotto, M. H. Szymanska, and N.P. Proukakis, Non-equilibrium Berezinskii-Kosterlitz-Thouless transition in driven-dissipative condensates (a), Europhys. Lett. 133, 17002 (2021).

[13] M. H. Szymanska, J. Keeling, and P. B. Littlewood, Meanfield theory and fluctuation spectrum of a pumped decaying Bose-Fermi system across the quantum condensation transition, Phys. Rev. B 75, 195331 (2007).

[14] G. Dagvadorj, J. M. Fellows, S. Matyjaśkiewicz, F. M. Marchetti, I. Carusotto, and M. H. Szymanska, Nonequilibrium Phase Transition in a Two-Dimensional Driven Open Quantum System, Phys. Rev. X 5, 041028 (2015).

[15] E. Altman, L. M. Sieberer, L. Chen, S. Diehl, and J. Toner, Two-Dimensional Superfluidity of Exciton Polaritons Requires Strong Anisotropy, Phys. Rev. X 5, 011017 (2015).

[16] A. Ferrier, A. Zamora, G. Dagvadorj, and M. H. Szymanska, Searching for the Kardar-Parisi-Zhang phase in microcavity polaritons, arXiv:2009.05177.

[17] P. Comaron, G. Dagvadorj, A. Zamora, I. Carusotto, N. P. Proukakis, and M.H. Szymanska, Dynamical Critical Exponents in Driven-Dissipative Quantum Systems, Phys. Rev. Lett. 121, 095302 (2018).

[18] A. Zamora, L. M. Sieberer, K. Dunnett, S. Diehl, and M. H. Szymanska, Tuning across Universalities with a Driven Open Condensate, Phys. Rev. X 7, 041006 (2017).

[19] A. L. Schawlow and C. H. Townes, Infrared and optical masers, Phys. Rev. 112, 1940 (1958).

[20] J. Kasprzak, M. Richard, S. Kundermann, A. Baas, P. Jeambrun, J. M. J. Keeling, F. M. Marchetti, M. H. Szymanska, R. Andre, J. L. Staehli, V. Savona, P. B. 
Littlewood, B. Deveaud, and L. S. Dang, Bose-Einstein condensation of exciton polaritons, Nature (London) 443, 409 (2006).

[21] K. S. Daskalakis, S. A. Maier, R. Murray, and S. KénaCohen, Nonlinear interactions in an organic polariton condensate, Nat. Mater. 13, 271 (2014).

[22] J. D. Plumhof, T. Stöferle, L. Mai, U. Scherf, and R. F. Mahrt, Room-temperature Bose-Einstein condensation of cavity exciton-polaritons in a polymer, Nat. Mater. 13, 247 (2014).

[23] I. Carusotto and C. Ciuti, Quantum fluids of light, Rev. Mod. Phys. 85, 299 (2013).

[24] J. Keeling and S. Kena-Cohen, Bose-Einstein condensation of exciton-polaritons in organic microcavities, Annu. Rev. Phys. Chem. 71, 435 (2020).

[25] Y. Sun, P. Wen, Y. Yoon, G. Liu, M. Steger, L. N. Pfeiffer, K. West, D. W. Snoke, and K. A. Nelson, Bose-Einstein Condensation of Long-Lifetime Polaritons in Thermal Equilibrium, Phys. Rev. Lett. 118, 016602 (2017).

[26] G. Roumpos, M. Lohse, W. H. Nitsche, J. Keeling, M. H. Szymanska, P. B. Littlewood, A. Löffler, S. Hofling, L. Worschech, A. Forchel, and Y. Yamamoto, Power-law decay of the spatial correlation function in exciton-polariton condensates, Proc. Natl. Acad. Sci. U.S.A. 109, 6467 (2012).

[27] W. H. Nitsche, N. Y. Kim, G. Roumpos, C. Schneider, M. Kamp, S. Hofling, A. Forchel, and Y. Yamamoto, Algebraic order and the Berezinskii-Kosterlitz-Thouless transition in an exciton-polariton gas, Phys. Rev. B 90, 205430 (2014).

[28] F. Baboux, D. D. Bernardis, V. Goblot, V. N. Gladilin, C. Gomez, E. Galopin, L. L. Gratiet, A. Lemaître, I. Sagnes, I. Carusotto, M. Wouters, A. Amo, and J. Bloch, Unstable and stable regimes of polariton condensation, Optica 5, 1163 (2018).

[29] A. P. D. Love, D. N. Krizhanovskii, D. M. Whittaker, R. Bouchekioua, D. Sanvitto, S. A. Rizeiqi, R. Bradley, M. S. Skolnick, P. R. Eastham, R. Andre, and L. S. Dang, Intrinsic Decoherence Mechanisms in the Microcavity Polariton Condensate, Phys. Rev. Lett. 101, 067404 (2008).

[30] H. Haug, T. D. Doan, H. T. Cao, and D. B. Tran Thoai, Temporal first- and second-order correlations in a polariton condensate, Phys. Rev. B 85, 205310 (2012).

[31] D. M. Whittaker and P. R. Eastham, Coherence properties of the microcavity polariton condensate, Europhys. Lett. 87, 27002 (2009).

[32] R. Spano, J. Cuadra, C. Lingg, D. Sanvitto, M. D. Martin, P. R. Eastham, M. v. d. Poel, J. M. Hvam, and L. Viña, Build up of off-diagonal long-range order in microcavity excitonpolaritons across the parametric threshold, Opt. Express 21, 10792 (2013).

[33] A. Clauset, C. R. Shalizi, and M. E. J. Newman, Power-law distributions in empirical data, SIAM Rev. 51, 661 (2009).

[34] M. De Giorgi, M. Ramezani, F. Todisco, A. Halpin, D. Caputo, A. Fieramosca, J. Gomez-Rivas, and D. Sanvitto, Interaction and coherence of a plasmon-exciton polariton condensate, ACS Photonics 5, 3666 (2018).

[35] The condensate size $500 \mu \mathrm{m}$ is 20 times the spatial coherence length of the polaritons in the uncondensed system (below the first threshold, $22 \mu \mathrm{m}$ [36]) and 100 times the healing length $\xi_{0}=\hbar / \sqrt{2 m_{\text {eff }} g n}=0.6, \ldots, 6 \mu \mathrm{m}$, where $m_{\mathrm{eff}}=1 e^{-7}-1 e^{-5} m_{e}$ and $g n=0.02 \mathrm{eV}$ [41].
[36] See Supplemental Material at http://link.aps.org/ supplemental/10.1103/PhysRevLett.127.255301 for detailed methodology and additional experiments, which includes Refs. [37-40].

[37] P. B. Johnson and R. W. Christy, Optical constants of noble metals, Phys. Rev. B 6, 4370 (1972).

[38] P. Törmä and W. L. Barnes, Strong coupling between surface plasmon polaritons and emitters: A review, Rep. Prog. Phys. 78, 013901 (2015).

[39] K. S. Daskalakis, Room-temperature polariton condensates in all-dielectric microcavities, Ph.D. thesis, Imperial College London, 2014.

[40] M. Born and E. Wolf, Principles of Optics (Pergamon Press, Oxford, 1998).

[41] A. I. Väkeväinen, A. J. Moilanen, M. Nečada, T. K. Hakala, K. S. Daskalakis, and P. Törmä, Sub-picosecond thermalization dynamics in condensation of strongly coupled lattice plasmons, Nat. Commun. 11, 3139 (2020).

[42] T. K. Hakala, A. J. Moilanen, A. I. Väkeväinen, R. Guo, J.-P. Martikainen, K. S. Daskalakis, H. T. Rekola, A. Julku, and P. Törmä, Bose-Einstein condensation in a plasmonic lattice, Nat. Phys. 14, 739 (2018).

[43] W. Wang, M. Ramezani, A. I. Väkeväinen, P. Törmä, J. G. Rivas, and T.W. Odom, The rich photonic world of plasmonic nanoparticle arrays, Mater. Today 21, 303 (2018).

[44] V. G. Kravets, A. V. Kabashin, W. L. Barnes, and A. N. Grigorenko, Plasmonic surface lattice resonances: A review of properties and applications, Chem. Rev. 118, 5912 (2018).

[45] D. Wang, W. Wang, M. P. Knudson, G. C. Schatz, and T. W. Odom, Structural engineering in plasmon nanolasers, Chem. Rev. 118, 2865 (2018).

[46] T. K. Hakala, H. T. Rekola, A. I. Väkeväinen, J.-P. Martikainen, M. Nečada, A. J. Moilanen, and P. Törmä, Lasing in dark and bright modes of a finite-sized plasmonic lattice, Nat. Commun. 8, 13687 (2017).

[47] K. S. Daskalakis, A. I. Väkeväinen, J.-P. Martikainen, T. K. Hakala, and P. Törmä, Ultrafast pulse generation in an organic nanoparticle-array laser, Nano Lett. 18, 2658 (2018).

[48] In this case, coherences may be present in the system density matrix even if the photon populations followed a thermal distribution.

[49] T. B. Hoang, G. M. Akselrod, A. Yang, T. W. Odom, and M. H. Mikkelsen, Millimeter-scale spatial coherence from a plasmon laser, Nano Lett. 17, 6690 (2017).

[50] S. Kim, B. Zhang, Z. Wang, J. Fischer, S. Brodbeck, M. Kamp, C. Schneider, S. Hofling, and H. Deng, Coherent Polariton Laser, Phys. Rev. X 6, 011026 (2016).

[51] T. Halpin-Healy and G. Palasantzas, Universal correlators and distributions as experimental signatures of $(2+1)$ dimensional Kardar-Parisi-Zhang growth, Europhys. Lett. 105, 50001 (2014).

[52] A. Pagnani and G. Parisi, Numerical estimate of the KardarParisi-Zhang universality class in $(2+1)$ dimensions, Phys. Rev. E 92, 010101(R) (2015).

[53] V. G. Miranda and F. D. A. Aarao Reis, Numerical study of the Kardar-Parisi-Zhang equation, Phys. Rev. E 77, 031134 (2008).

[54] L. He, L. M. Sieberer, E. Altman, and S. Diehl, Scaling properties of one-dimensional driven-dissipative condensates, Phys. Rev. B 92, 155307 (2015). 
[55] R. Moerland, T. Hakala, J.-P. Martikainen, H. Rekola, A. Väkeväinen, and P. Törmä, Strong coupling between organic molecules and plasmonic nanostructures, in Quantum Plasmonics (Springer, Cham, 2017).

[56] R. Guo, T. K. Hakala, and P. Törmä, Geometry dependence of surface lattice resonances in plasmonic nanoparticle arrays, Phys. Rev. B 95, 155423 (2017).
[57] J. Keeling, L. M. Sieberer, E. Altman, L. Chen, S. Diehl, and J. Toner, Superfluidity and phase correlations of driven dissipative condensates, in Universal Themes of Bose-Einstein Condensation (Cambridge University Press, Cambridge, England, 2017), pp. 205230. 